\title{
science \\ Self-promotions and advertising: are they a common practice for boosting altmetric scores?
}

\author{
Sai Krishna Gudi', Swarna Priya Basker² \\ ${ }^{1}$ College of Pharmacy, Rady Faculty of Health Sciences, University of Manitoba, Winnipeg, MB, Canada; ' Jaya College of \\ Pharmacy, Chennai, India
}

\section{Introduction}

In the era of internet and social media, the modes of publishing, sharing, searching, and reading scientific research are evolving, as the ultimate goal of any research is to be discoverable to the target audience. Altmetrics is a form of qualitative data that are complementary to traditional, citation-based metrics, which deal with journal articles and other scholarly outputs being discussed worldwide [1]. In recent times, altmetrics has gained reputation and popularity as it offers a quicker way to demonstrate the potential impact of one's scholarly work and its public engagement. Although enhancing research impact and gaining visibility are essential from a researcher's viewpoint, self-advertising should be prudent and appropriate. Through this essay, we intend to discuss on how self-promotions and advertising play an active role in driving altmetric scores.

\section{Definition of Altmetrics}

The term altmetrics is derived from the terms 'alternative' and 'metrics', and measures the interactions among researchers, academicians, scholars, and scientists that are captured by social media such as Facebook, Twitter, LinkedIn, and blogs, and by specific reference management tools such as Mendeley [1]. Typically, for an article-level metrics, a multi-colored altmetric donut badge icon is found alongside each article with a number at the center. This number is de-

Received: June 25, 2019

Accepted: July 1, 2019

Correspondence to Sai Krishna Gudi gudisk@myumanitoba.ca

ORCID

Sai Krishna Gudi

https://orcid.org/0000-0001-9576-9207

Swarna Priya Basker

https://orcid.org/0000-0002-1877-3808 noted as the Altmetric Attention Score or Altmetric Score (AS) for the article [2]. AS was first developed and established by Euan Adie in 2010 and came into use in the United States, United Kingdom, Germany, and Australia in 2012 [3]. Traditionally, research capability and intelligence were measured using citation-based metrics; however, as citations cannot provide information on downloads, mentions, and shares, which have come into existence after the development and evolution of the Web, AS came into action. 


\section{Functions of AS}

Traditional scholarly metrics such as journal impact factor (JIF) are often used as an evaluation tool to appraise publications of individual researchers. Citations are the established metric for impact evaluation and are more meaningful than the metrics of the journal [4]. Further, the h-index is a popular metric to quantify an individual's scientific research output. However, AS can track the number of views, downloads, bookmarks, shares, and mentions of a published paper in policy documents, press releases, news outlets, and references in Wikipedia [5]. Thus, these scores have become undoubtedly crucial to researchers given that they measure dissemination, and act as an indicator of influence and impact of their published scholarly work. AS considers and measures various parameters such as individual collaboration, international collaboration, institution impact, JIF, journal open accessibility, and field type when generating scores [6].

The ultimate goal of any research is to promote and showcase the findings to reach the target audience, which could, in turn, enhance the reputation and career progression of a researcher. Online media channels, including blogging sites and the mass social media networks such as Twitter, have soon become essential communication channels for scientists to generate and discuss ideas, find collaborators, and disseminate research both within their communities and to the general public [7]. As AS's provide an initial and immediate assessment of a research article's future scholarly impact, researchers are inclined toward social media, which is freely available and convenient for engagement. However, the citations of an article cannot be predicted based on AS. The number of times an article is discussed on the altmetric platform is an essential indicator of its impact and contribution to the research world. AS is useful to rank research outputs based on attention gained by that particular article; however, it cannot estimate anything about research quality [8]. Promoting research on social media can play a vital role in gaining visibility, which eventually may improve AS, and thus gain a more diverse impact than that achieved by citation-based metrics; further, it may analyze the societal impact of certain scholarly publications. However, AS is relatively new and more research is required for their use. Even when a publication lacks citations, AS can act as a potential measure of the value of research outputs, thereby providing an independent assessment of research engagement with the public and their feedback. However articles can sometimes receive online attention for the wrong reasons. For example, if an article contains flaws or any surrounding controversies and is then discussed on social media, the article would still receive a high AS for inaccurate work. Moreover, discussions and mentions can be purchased to increase a paper's AS, and publishers could also collaborate with specific news agencies to increase the coverage of their research articles by the mainstream media [9].

\section{Ways to Publicize Scholarly Work}

Social media enables one to share ideas and receive an immediate response to the sharing activities. Because of its quick response and efficiency, it has attracted the attention of the scientific community in parallel to the traditional forms of scholarly communication. With the presence of social media, newer articles have an inherent advantage of having a higher AS over older ones. There are different ways in which individuals can publicize their work online, e.g., tweeting, which is the most significant contributor to altmetrics, posting on Facebook and LinkedIn, blogging, and adding references of published works to Wikipedia and other public information platforms [10]. At times, even journals promote their published articles on social media for greater outreach, engagement and improved dissemination. Nowadays, a formal publication of scholarly work in a peer-reviewed journal is not considered to be the final step, and publicizing and promoting the published work, which are potentially beneficial to the target population, science community, and the broader society, are treated to be equally important. The tools under the umbrella of altmetrics allow researchers to move out from the traditional closed system to the open Web to share their ideas and findings; receive comments; and get their research referenced and peer-reviewed from a wide range of diversified users. However, not everyone will encourage such promotions and respond positively to it. Moreover, these may also create unwanted noise on the Web.

\section{Complementary Roles of Altmetrics to Bibliometrics}

From a recent survey, there are approximately 10.9 million mentions of new stories till date in the metadata of journals, and a majority of the attention was noticed after the emergence of AS (i.e., after 2012), which represents an increase in self-promotions of research on social media [3]. Presence of a positive correlation between social media mentions and future citations suggest that online activity may anticipate the traditional measure of scholarly impact [6]. Thus, AS and online activity have the potential to boost future citation rates. However, altmetrics are a complement to, and not a replacement for, factors like informed peer review and citation-based metrics [11]. Findings from a recent study suggest that in countries with limited access to the internet and social media services, the utility and reach of altmetrics may be limited, indicating a clear-cut dependence of AS on social media [12]. 
As a young discipline, altmetrics is quickly changing the dynamics and incentives of scholarly communication and scientific publishing. However, it should not replace traditional bibliometrics (citations, JIF, and h-index), but rather supplement them. Criticizing and discussing a quality research is important, rather than boosting AS's. Although self-promotions and advertising on social-media are linked with the high AS's and citation rates, researchers should act prudently and appropriatly when publicizing their scholarly work to avoid unwanted noise on the web.

\section{Conclusion}

Through all these explorations, it is evident that social media has a definitive impact on AS promotion, which is, in fact, a common approach of most researchers to uphold their reputation. However, we must wait and watch how this association between social media and article metrics changes scholarly communication and science itself in the near future.

\section{Conflict of Interest}

No potential conflict of interest relevant to this article was reported.

\section{References}

1. Rosenkrantz AB, Ayoola A, Singh K, Duszak R Jr. Alternative metrics for assessing article impact in popular general radiology journals. Acad Radiol 2017;24:891-7. https://doi. org/10.1016/j.acra.2016.11.019

2. Piwowar H. Altmetrics: value all research products. Nature 2013;493:159. https://doi.org/10.1038/493159a

3. Konkiel S. 5 Things you may not know about Altmetric's altmetrics [Internet]. London: Altmetric; 2019 [cited 2019
May 26] Available from: https://www.altmetric.com/blog/5things-you-may-not-know-about-altmetrics-altmetrics/

4. Garfield E. Citation analysis as a tool in journal evaluation. Science 1972;178:471-9. https://doi.org/10.1126/science.178.4060.471

5. Amath A, Ambacher K, Leddy JJ, Wood TJ, Ramnanan CJ. Comparing alternative and traditional dissemination metrics in medical education. Med Educ 2017;51:935-41. https://doi.org/10.1111/medu.13359

6. Brown A. Research metrics: altmetrics [Internet]. Advanced Science News; 2019 [cited 2019 May 26]. Available from: https://www.advancedsciencenews.com/researchmetrics-altmetrics

7. Eysenbach G. Can tweets predict citations? Metrics of social impact based on Twitter and correlation with traditional metrics of scientific impact. J Med Internet Res 2011;13: e123. https://doi.org/10.2196/jmir.2012

8. Khodiyar VK, Rowlett KA, Lawrence RN. Altmetrics as a means of assessing scholarly output. Learn Publ 2014;27: S25-32. https://doi.org/10.1087/20140505.

9. Thelwall M, Haustein S, Lariviere V, Sugimoto CR. Do altmetrics work? Twitter and ten other social web services. PLoS One 2013;8:e64841. https://doi.org/10.1371/journal. pone.0064841

10. Thoma B, Joshi N, Trueger NS, Chan TM, Lin M. Five strategies to effectively use online resources in emergency medicine. Ann Emerg Med 2014;64:392-5. https://doi. org/10.1016/j.annemergmed.2014.05.029

11. Warren HR, Raison N, Dasgupta P. The rise of altmetrics. JAMA 2017;317:131-2. https://doi.org/10.1001/jama.2016.18346.

12. Trueger NS, Thoma B, Hsu CH, Sullivan D, Peters L, Lin M. The Altmetric Score: a new measure for article-level dissemination and impact. Ann Emerg Med 2015;66:54953. https://doi.org/10.1016/j.annemergmed.2015.04.022 